
ISSN 1813-548X

\title{
Influence thermique de l'emplacement du toit en chaume sous le toit en tôle d'un habitat à Antananarivo- Madagascar
}

\section{Solofonirina ROBELISON ${ }^{1 *}$ et Bernard LIPS ${ }^{2}$}

1Institut pour la Maîtrise de l'Energie. Université d'AntananarivoMadagascar

2Université de Lyon, CETHIL UMR5008, INSA de Lyon, CNRS, Université Lyon 1, Centre Thermique de Lyon, 20, avenue Albert Einstein, 69621, Villeurbanne CEDEX, France

*Correspondance, courriel : robelison_60@yahoo.fr

\section{Résumé}

Une technique de rafraîchissement thermique du grenier d'une maison à Antananarivo (Madagascar) a été étudiée pendant la période chaude et humide. Ventilation du grenier ou isolation du toit en tôle a été envisagée. La température de l'air dans le grenier a été déterminée pendant 24 heures en prenant le jour du 21 janvier pour chacune de ces deux techniques. Au maximum, une diminution de $2,5^{\circ} \mathrm{C}$ pour la ventilation et une diminution de température de $16^{\circ} \mathrm{C}$ pour l'isolation par le chaume ont été obtenues. C'est l'isolation du toit en tôle par un isolant qui est la meilleure technique de rafraîchissement.

Mots-clés : Maison, ventilation, isolation, rafraîchissement, Madagascar.

\section{Abstract}

Influence thermal site of the thatched roof under the sheet roof of a habitat with Antananarivo- Madagascar

A thermal technique of cooling of the attic of a house with Antananarivo (Madagascar) was studied for the hot and wet period. Ventilation of the 
attic or insulation of the sheet roof was considered. The temperature of the air in the attic was given during 24 hours by taking the day of January 21 for each one of these two techniques. To the maximum, a reduction in $2,5^{\circ} \mathrm{C}$ for ventilation and a reduction in temperature of $16^{\circ} \mathrm{C}$ for the insulation by thatch were obtained. It is the insulation of the sheet roof by an insulator which is the best technique of cooling.

Keywords : House, ventilation, insulation, cooling, Madagascar.

\section{Introduction}

Le toit en chaume, connu pour sa haute qualité d'isolant thermique, est utilisé depuis longtemps dans les villages des hautes Terres Centrales à Madagascar. Ce type de toit présente cependant une mauvaise qualité d'étanchéité. Actuellement, le chaume est remplacé par de la tôle moins coûteuse et possédant une très bonne qualité d'étanchéité. Sa bonne conductivité thermique dégrade le confort thermique de la maison pendant la saison chaude et humide. Dans cet article, nous étudions le comportement thermique du grenier d'une maison- type en comparant les deux types de toiture. Deux solutions (ventilation du grenier ou isolation) sont étudiées pour améliorer le comportement thermique du toit en tôle. Les hypothèses adoptées sont les suivantes : Le toit est supposé plan et uniforme quelque soit sa nature (tôle ou chaume), le plafond est supposé plan et uniforme, l'air du volume intérieur du grenier est supposé isotherme, les inerties des murs au Nord et au Sud sont négligées, les effets de bord sont négligeables, le plafond, séparant le grenier et le 1er étage, est supposé plan et uniforme, l'échange thermique par conduction des chevrons, supports du plafond et du plancher du $1^{\text {er }}$ étage, est négligeable, la température de l'air dans le 1er étage est supposée égale à celle de l'air extérieur. Ceci est valable quand les fenêtres et les portes du $1^{\text {er }}$ étage sont ouvertes. Le schéma analogique thermique- électrique a été utilisé pour la mise en équations du problème et les températures inconnues sont déterminées par une équation matricielle en utilisant la méthode de KHALETSKI. Un de ces systèmes est meilleur quand la 
température de l'air dans le grenier est plus près de celle du 1er étage pendant une journée.

\section{Matériel et méthodes}

\section{2-1. Site d'étude}

La maison étudiée se trouve dans le village de Botona (commune de Sabotsy- Namehana) à $12 \mathrm{~km}$ au nord d'Antananarivo. II existe trois types de toits à Botona : toit en chaume ( $25 \%$ des maisons), toit en tuiles artisanales ( $5 \%$ des maisons) et toit en tôle ondulée ou galvabac (70 $\%$ ). La maison étudiée est implantée sur un terrain plat de $600 \mathrm{~m}^{2}$ en site isolé. A l'ouest des rizières transformées en carrière de briqueteries s'étalent jusqu'à une colline. Au sud, à l'Est et au Nord, les maisons les plus proches se trouvent à une dizaine de mètres (Figure1).

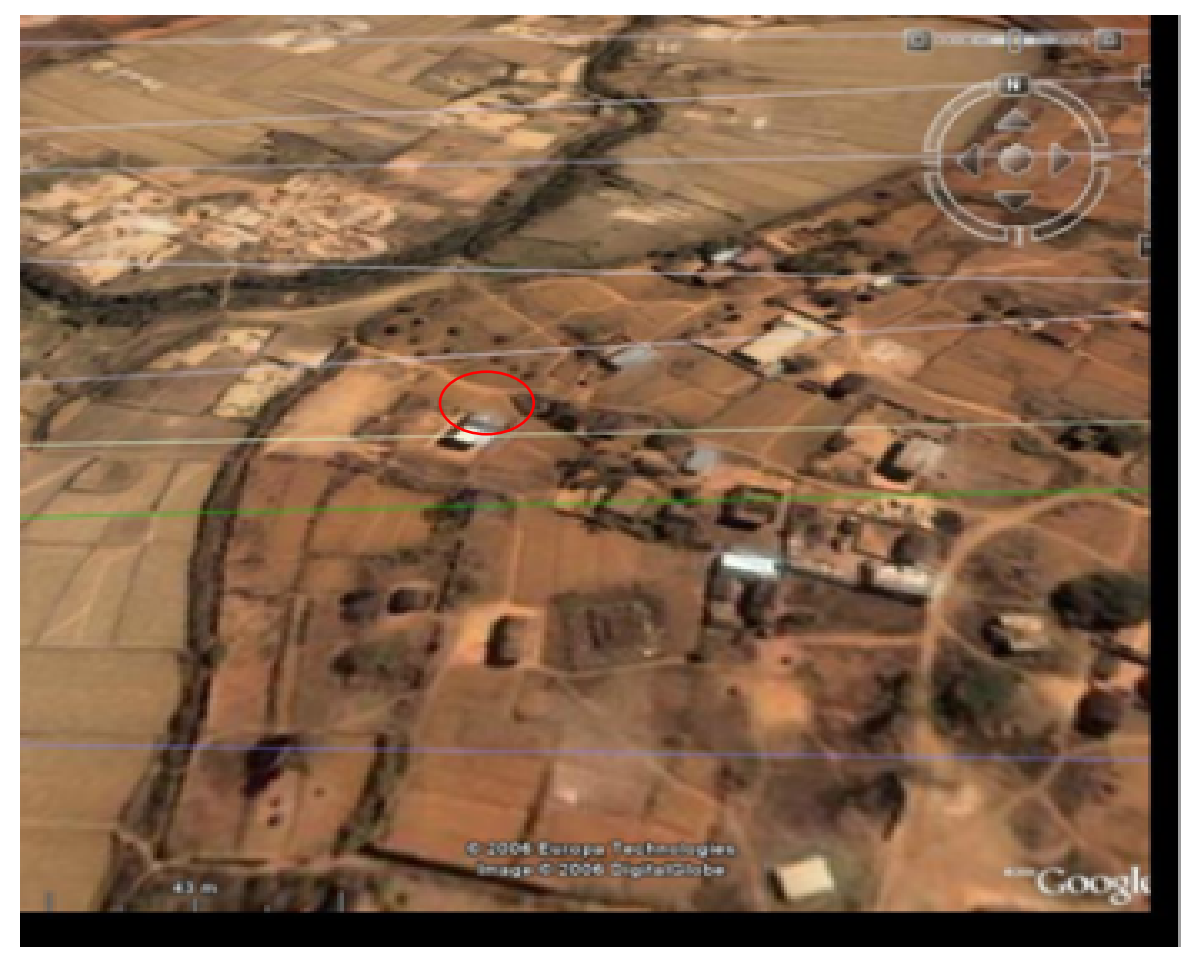

Figure 1 : Emplacement du bâtiment à étudier 
Ce bâtiment se trouve à la latitude de $18^{\circ} 50^{\prime} 01^{\prime \prime}$ 's à la longitude de $47^{\circ} 34^{\prime} 18^{\prime \prime} \mathrm{E}$ et à l'altitude de $1264 \mathrm{~m}$. La saison chaude et humide correspond aux mois d'octobre à mars (été) et la saison sèche et froide (hiver) aux mois d'avril à septembre. La maison est un bâtiment à un étage de $10 \mathrm{~m}$ de long extérieur pour $7 \mathrm{~m}$ de large extérieur. (Figure 2).



Figure 2 : Vue cavalière de la maison

Il comporte :

- À l'Ouest : six ouvertures dont deux fenêtres aux rez-de-chaussée, et une grand véranda.

- Au nord : quatre fenêtres dont deux aux rez-de-chaussée et deux au premier étage.

- Au sud et à l'Est, pas d'ouvertures.

Les fenêtres sont en bois de chêne d'épaisseur de $2 \mathrm{~cm}$ sans vitrage. Ces fenêtres sont totalement ouvertes pendant le jour.

L'épaisseur du mur est de $33 \mathrm{~cm}$. Les murs sont construits en briques d'argile cuite $11 \times 11 \times 22 \mathrm{~cm}$, de masse volumique de $1950 \mathrm{~kg} / \mathrm{m}^{3}$. Le mur, à l'extérieur et à l'intérieur, est recouvert d'une chape de terre battue.

Le toit en tôle est incliné de $30^{\circ}$ par rapport à l'horizontale. Le toit au dessus de la véranda de $10^{\circ}$. Ces toits sont soutenus par des bois ronds de $4 \mathrm{~m}$ de long et de $8 \mathrm{~cm}$ de diamètre.

Le plancher du premier étage est un parquet en bois de chêne (Lames de 2 $\mathrm{cm}$ d'épaisseur, de $8 \mathrm{~cm}$ de large pour $4 \mathrm{~m}$ de long). Le plancher est 
soutenu par des madriers de $3 \mathrm{~m}$ de long, $12 \mathrm{~cm}$ de large et de $5 \mathrm{~cm}$ de hauteur.

II n'y a pas de sous- sol. Le sol du rez-de-chaussée est une chape de ciment de $3 \mathrm{~cm}$ d'épaisseur.

Tout autour du bâtiment, la cour est un sol en latérite rouge.

Nombre d'occupants permanents : 2 adultes et 4 enfants.

La maison comporte cinq chambres au rez-de-chaussée et trois grandes chambres au $1^{\text {er }}$ étage dont nous présentons le plan du rez - de chaussée (Figure 3) et celui du 1er étage_(Figure 4).

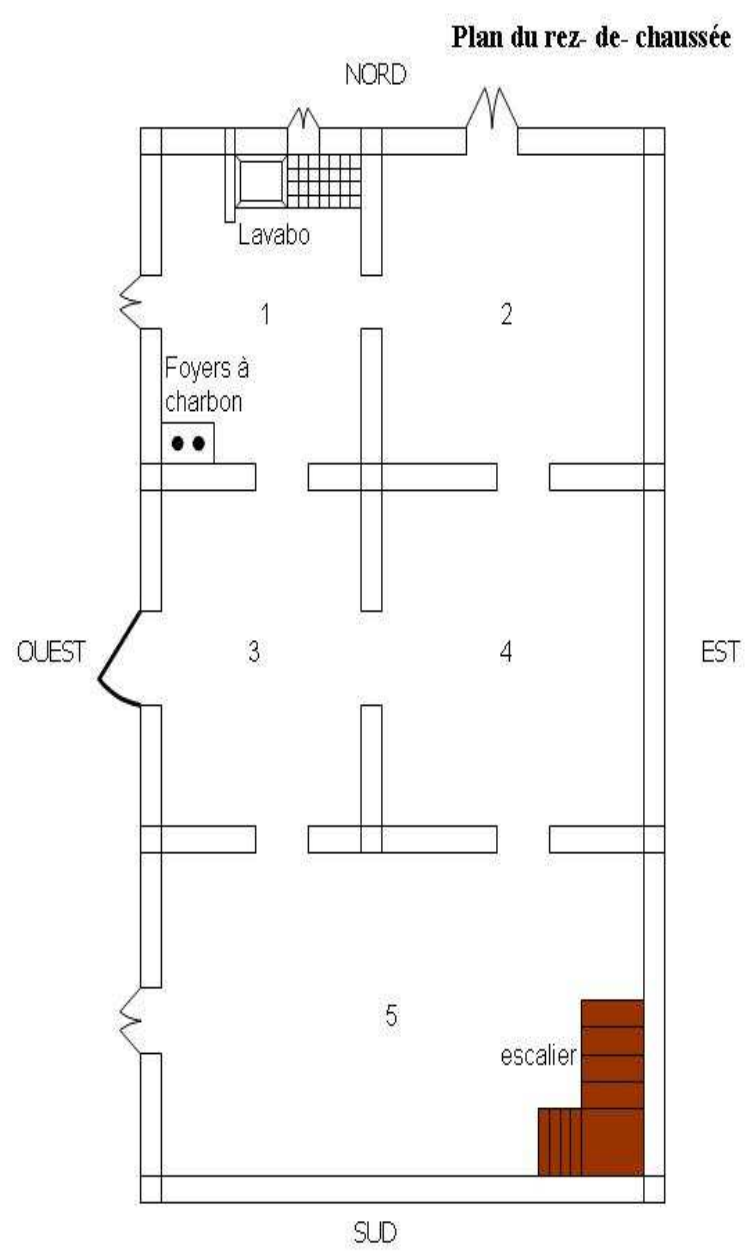

1: cuisine

2: chambre à coucher

3: chambre vide $n^{\circ} 1$

4. chambre vide $n^{\circ} 2$

5: salon

Légende :

Fenêtre à double battant

Porte à simple battant

Figure 3 : Plan du rez - de - chaussée 


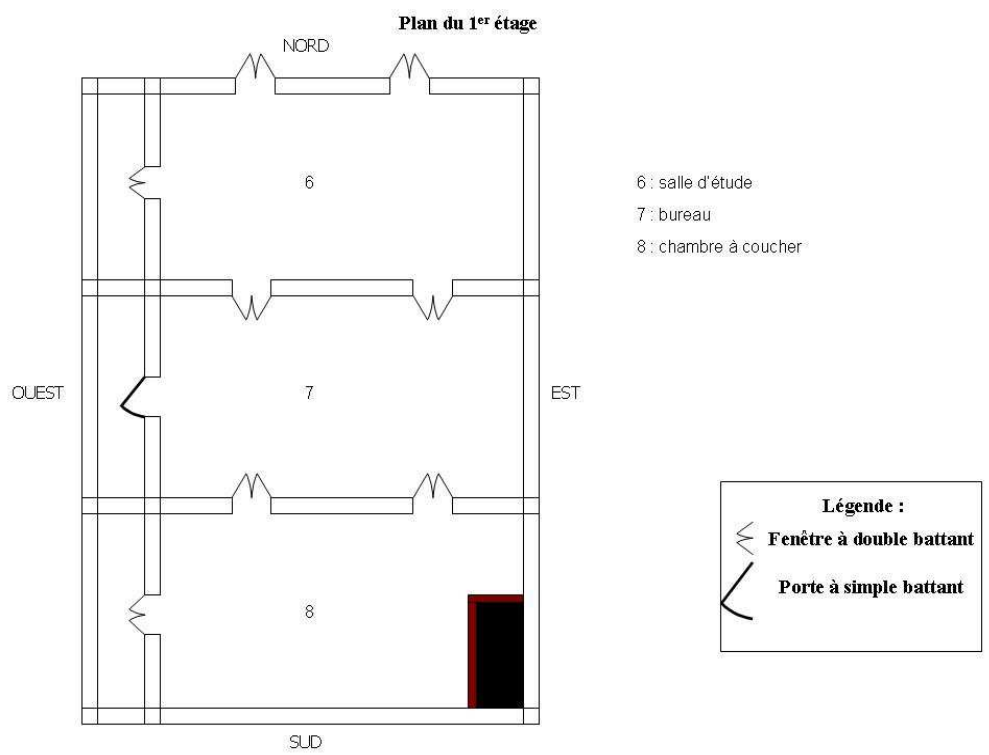

Figure 4 : Plan du 1er étage

Chambre $1:$ II y a deux foyers à charbon de bois, un lavabo et une petite douche dans la cuisine. Elle n'a pas encore de cheminée par conséquent, la fumée se répand rapidement dans la chambre 2. Elle reçoit le rayonnement solaire provenant des fenêtres au Nord et à l'Ouest.

Chambre 2 : C'est une chambre à coucher pour deux personnes adultes. Elle comporte essentiellement une petite bibliothèque et un poste de télévision. Elle reçoit du rayonnement solaire provenant de la fenêtre au Nord.

Chambres 3 et 4 : Elles sont totalement obscures. Ce sont actuellement des chambres non utilisées. Elles ne reçoivent pas de rayonnements solaires.

Chambre 5 : Elle sert de salon. II y a un escalier menant vers le premier étage. Il y a des meubles, un poste de télévision, et un lit superposé.

Chambre 6: elle sert de bureau aux quatre enfants. Elle reçoit convenablement de la lumière provenant des deux fenêtres Nord et de celle de l'Ouest.

Chambre 7 : Elle sert de bureau de travail. 
Chambre 8: c'est une chambre à coucher contenant deux lits et quelques meubles. Elle reçoit de la lumière l'après-midi.

\section{2-2. Analyses}

Le modèle thermique développé ne concerne que le grenier et prend en compte les divers flux thermiques entre le grenier et le milieu extérieur ainsi qu'entre le grenier et le premier étage.

\section{2-2-1. Différents modes d'échanges thermiques}

La figure 5 schématise les divers flux thermiques pris en compte par le modèle:

* rayonnement du soleil chauffant la surface extérieure du toit. $\mathrm{Ce}$ rayonnement varie tout au long de la journée.

* rayonnement du toit vers le ciel (grande longueur d'onde)

* échange thermique par convection entre la surface du toit et l'air extérieur

* conduction thermique dans le matériau du toit et dans l'éventuel isolant

* échanges par convection entre la surface intérieure du toit et l'air du grenier.

* échanges par convection et conduction entre l'air du grenier et le premier étage de la maison

* flux thermique du à la ventilation du grenier 


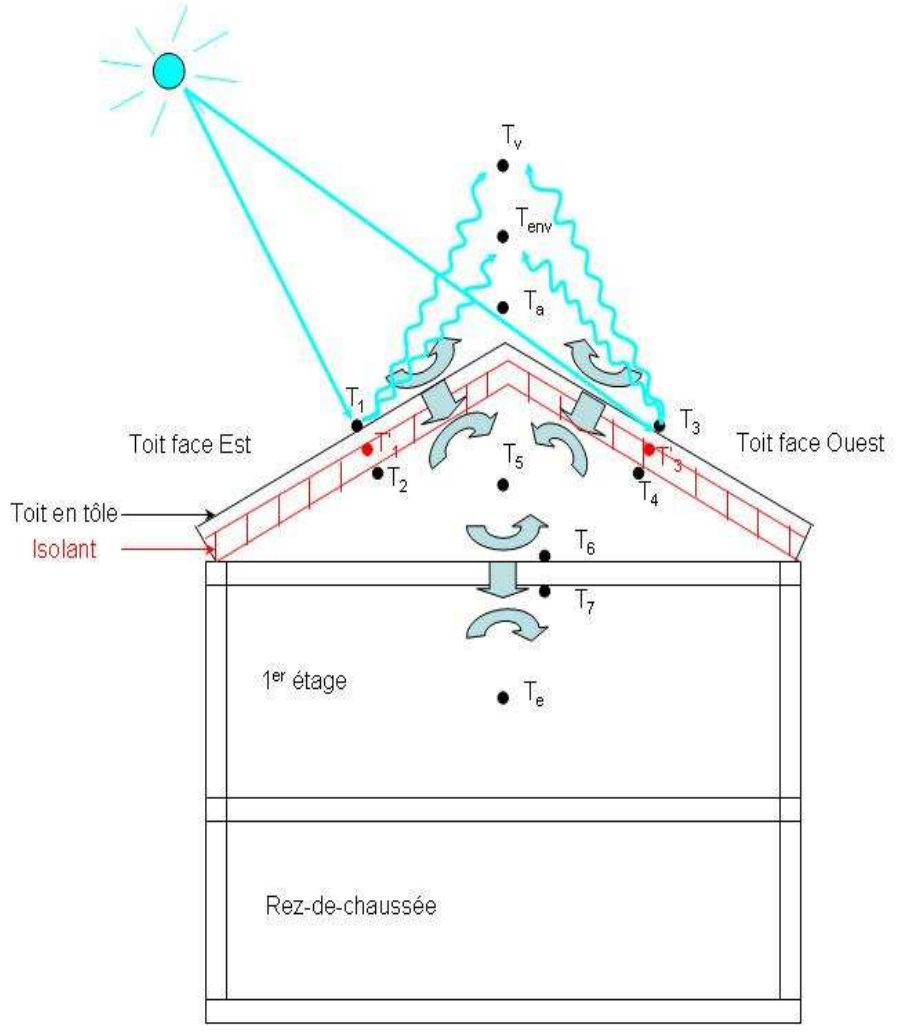

Figure 5 : Différents modes d'échanges thermiques entre le grenier et le 1er étage

\section{2-2-2. Formulations des échanges thermiques}

Echange thermique par rayonnement

- Rayonnement global reçu par le plan incliné []

La densité de rayonnement global Gp reçu par un plan incliné est la somme de celles du rayonnement direct $I_{p}$, du rayonnement diffus $D_{p}$ et du rayonnement réfléchi $\mathrm{R}_{\mathrm{P}}$.

$$
G_{P}=I_{P}+D_{P}+R_{P}
$$

Les flux thermiques de courte longueur d'onde reçu par la surface sont égaux au produit du rayonnement global $G_{p}$ par la surface $S$ du toit.

$$
\Phi_{C L}=G_{P} . S
$$


Remarque :

Le calcul de Gp nécessite la connaissance des valeurs du rayonnement direct $I_{H}$ et du rayonnement diffus $D_{H}$ ainsi que celui du rayonnement global $G_{H}$ reçus par un plan horizontal. Ces valeurs de $I_{H}$ et de $D_{H}$ sont données par les fichiers météorologiques. Ainsi $\mathrm{G}_{H}$ se calcule aisément par la relation :

$$
G_{H}=I_{H}+D_{H}
$$

- Echange de rayonnement de grande longueur d'onde (GLO) :

II y a échange de rayonnement de grande longueur d'onde entre la face supérieure du toit et la voûte céleste d'une part, et entre la face supérieure du toit et l'environnement d'autre part. L'échange entre la face supérieure du toit et la voûte céleste est donné par :

$\Phi_{T V}=h_{v} \cdot S\left(T_{\text {toit }, e x t}-T_{v}\right)$

Avec :

$$
h_{v}=\sigma \varepsilon \frac{1+\cos \alpha}{2}\left(T_{v}{ }^{2}+T_{\text {toit }, e x t}{ }^{2}\right)\left(T_{v}+T_{\text {toit }, \text { ext }}\right)
$$

L'échange entre la face supérieure du toit et l'environnement est donné par:

$\Phi_{T E}=h_{\text {env }} . S\left(T_{\text {toit }, e x t}-T_{\text {env }}\right)$

Avec :

$$
h_{e n v}=\sigma \varepsilon \frac{1-\cos \alpha}{2}\left(T_{e n v}{ }^{2}+T_{\text {toit }, e x t}{ }^{2}\right)\left(T_{e n v}+T_{\text {toit }, e x t}\right)
$$

$T_{\text {env }} \approx T_{a}$

Le flux thermique de grande longueur d'onde émis par le toit sera donc donné par :

$$
\Phi_{G L}=\Phi_{T V}+\Phi_{T E}
$$

$>$ Echange thermique par convection 
- Echange thermique par convection extérieur

Le flux thermique par convection extérieure, entre le vent et la face supérieure du toit, est donné par :

$$
\Phi_{\text {conv }, e x t}=h_{\text {conv }, \text { ext }} . S\left(T_{\text {toit }, \text { ext }}-T_{a}\right)
$$

Avec :

$$
h_{\text {conv }, \text { ext }}=5,7 \mathrm{~V}+11,4
$$

C'est la formule de STURROCK.

Nous ne tenons pas compte si le toit est sous le vent ou au vent.

- Echange thermique par convection intérieur

Le flux thermique par convection intérieure, entre l'air du grenier et la face inférieure du toit, est donné par :

$$
\Phi_{\text {conv,int }}=h_{\text {conv,int }} \cdot S\left(T_{\text {toit,int }}-T_{\text {Grenier }}\right)
$$

Le flux thermique par convection intérieure, entre le vent dans le grenier et la face supérieure du plancher, est donné par :

$$
\Phi_{\text {conv,sup } P L}=h_{\text {conv,sup }} . S^{\prime}\left(T_{\text {Grenier }}-T_{P L \text {,sup }}\right)
$$

Le flux thermique par convection intérieure, entre l'air du 1er étage et la face inférieure du plancher, est donné par :

$$
\Phi_{\text {conv }, \text { inf } P L}=h_{c o n v, \text { inf }} . S^{\prime}\left(T_{P L, \text { inf }}-T_{e}\right)
$$

$>$ Echange thermique par conduction

- Le flux thermique par conduction à travers le toit:

$$
\Phi_{\text {cond,toit }}=h_{\text {cond }, \text { toit }} \cdot S \cdot\left(T_{\text {toit }, \text { ext }}-T_{\text {toit }, \text { int }}\right)
$$

Avec : 


$$
h_{\text {condtoit }}=\frac{1}{\frac{e_{\text {toit }}}{\lambda_{\text {toit }}}+\frac{e_{\text {isolant }}}{\lambda_{\text {isolant }}}}
$$

$e_{i s o l a n t}=0$ si on ne met pas un isolant sous la face inférieure du toit.

- Le flux thermique par conduction à travers le plancher :

$$
\Phi_{\text {cond }, P L}=h_{\text {cond }, P L} \cdot S^{\prime} \cdot\left(T_{P L, \text { sup }}-T_{P L, \text { inf }}\right)
$$

Avec :

$$
h_{\text {cond }, P L}=\frac{\lambda_{P L}}{e_{P L}}
$$

\section{2-2-3. Inerties}

La capacité d'inertie d'un matériau mesure son aptitude à stocker la chaleur et à en différer la restitution.

- Capacité d'inertie du toit

Pour le toit sans isolant :

$$
C_{\text {toit }}=\rho_{\text {toit }} \cdot e_{\text {toit }} \cdot S \cdot C_{p, t o i t}
$$

Dans le cas où nous mettons un isolant au - dessous du toit, nous aurons deux capacités montées en parallèle: celle du toit et celle de l'isolant, dont la capacité équivalente est donnée par :

$$
C_{\text {toit, isolant }}=\rho_{\text {toit }} \cdot e_{\text {toit }} \cdot S \cdot C_{p, \text { toit }}+\rho_{\text {isolant }} \cdot e_{\text {isolant }} \cdot S \cdot C_{p, \text { isolant }}
$$

- Capacité d'inertie du plafond

Le plafond, qui sépare le grenier et le premier étage, a une capacité égale à :

$$
C_{P L}=\rho_{P L} \cdot e_{P L} \cdot S^{\prime} \cdot C_{P L}
$$




\section{2-2-4. Ventilation}

Le ventilateur d'entrée au Sud fait entrer de l'air à la température $T_{a}$ tandis que l'autre au Nord le fait sortir à la température TGrenier (Figure 2). L'énergie thermique dissipée par l'air de la ventilation est exprimée par :

$$
P_{u}=D_{e} \cdot C_{p a} \cdot\left(T_{\text {Grenier }}[t]-T_{a}[t]\right)
$$

On appelle taux de renouvellement d'air $\tau$ le nombre de volume d'air changé par la ventilation par unité de temps. II est égal au rapport du débit d'air par le volume de la zone. Par conséquent, le débit d'air est alors donné par:

$$
D_{e}=\tau . V_{a, \text { Grenier }}
$$

Dans le cas d'absence de ventilation, le taux de renouvellement d'air est nul. $\tau=0$

\section{Modélisation}

Le schéma électrique analogique (Figure 6) est basé sur le modèle 2RC c'est-à-dire deux résistances et une capacité.

Nous utilisons la loi de KIRCHOFF, la loi d'OHM et l'analogie entre thermique et électrique à chaque nœud de température pour établir l'équation différentielle qui régit l'échange thermique. La loi de KIRCHOFF dit que la somme des courants entrants est égale à celle des courants sortants pour chaque nœud tandis que la loi d'OHM dit que la tension (ou différence de potentiel) entre deux nœuds est égale au produit de l'intensité du courant par la résistance, et enfin _l'analogie thermique - électrique :

Courant électrique $\rightarrow$ Flux thermique ou inertie thermique

Différence de potentiel $\rightarrow$ Différence de températures

Résistance électrique $\rightarrow$ Résistance thermique

En utilisant la méthode de discrétisation vers l'arrière des équations différentielles en températures, nous obtenons un système de sept 
équations linéaires à sept inconnues suivant dont les inconnues sont les températures :

$T_{\text {toit,ext }} ; T_{\text {toit, int } E} ; T_{\text {toit, ext }} ; T_{\text {toit, int } W} ; T_{\text {Grenier }} ; T_{P L, \text { sup }} ; T_{P L \text {,inf }}$.

Sous forme matricielle, ce système d'équations s'écrit sous la forme :

$[A]\left[T^{t}\right]=\left[\frac{C}{\Delta t} T^{t-1}\right]+\left[U^{t}\right]$

Les inconnues s'obtiennent par l'équation matricielle :

$\left[T^{t}\right]=[A]^{-1} \cdot B$

Avec :

$B=\left[\frac{C}{\Delta t} T^{t-1}+U^{t}\right]$

t étant l'instant; $\mathrm{t}$ varie de $1 \mathrm{~h}$ à $24 \mathrm{~h}$.

Où $[A]^{-1}$ étant la matrice inverse de la matrice $[A]$, elle est obtenue en utilisant la méthode de KHALETSI. 


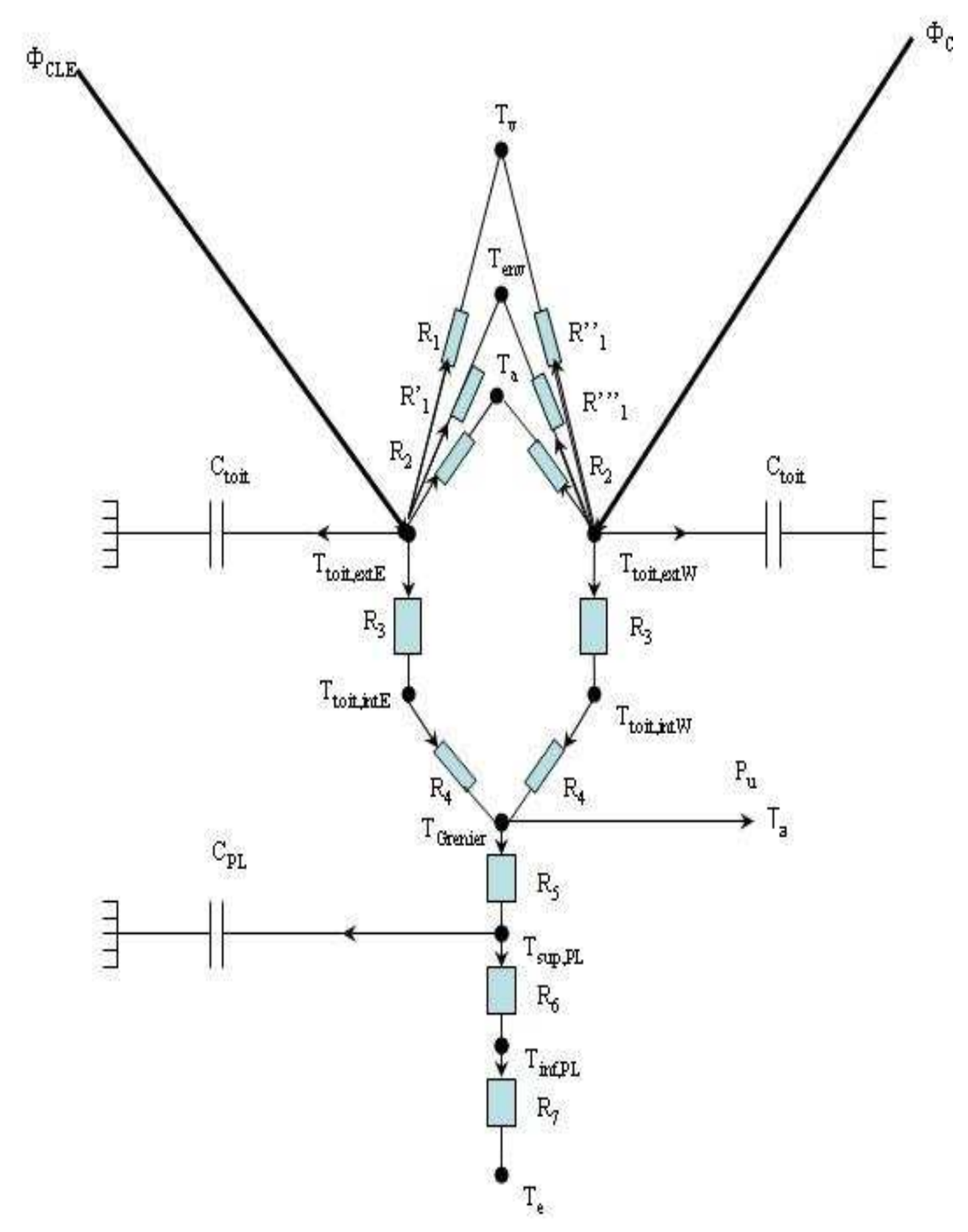

Schéma électrique analogique du grenier

Figure 6 : Schéma électrique analogique du grenier

Avant d'analyser les résultats obtenus après les calculs, précisons les données numériques utilisées pour la simulation (Tableau)

Tableau : Données numériques utilisées pour la simulation

\begin{tabular}{|l|l|}
\hline Grandeurs & Valeurs \\
\hline Albédo & 0,1 \\
Masse volumique du toit en tôle & $2787 \mathrm{~kg} / \mathrm{m}^{3}$ \\
Surface du toit face Est ou face Ouest & $35,30 \mathrm{~m}^{2}$ \\
\hline
\end{tabular}


Chaleur spécifique du toit en tôle

Conductivité thermique du toit en tôle

Angle d'inclinaison du toit

Epaisseur du toit en tôle

Masse volumique du plafond en pin

Epaisseur du plafond

Surface du plafond

Chaleur spécifique du plafond en pin

Volume de l'intérieur du grenier

Conductivité thermique du plafond en pin

Masse volumique du toit en chaume

Epaisseur du toit en chaume

Chaleur spécifique du toit en chaume

Conductivité thermique du toit en chaume

Masse volumique de l'ouate de coton

Chaleur spécifique de l'ouate de coton

Conductivité thermique de l'ouate de coton

\begin{tabular}{|ll|}
\hline $0,251 \mathrm{Wh} / \mathrm{kg} \cdot \mathrm{K}$ \\
163 & $\mathrm{~W} / \mathrm{m} \cdot \mathrm{K}$ \\
$30^{\circ}$ & \\
0,001 & $\mathrm{~m}$ \\
546 & $\mathrm{~kg} / \mathrm{m}^{3}$ \\
0,02 & $\mathrm{~m}$ \\
61,2 & $\mathrm{~m}^{2}$ \\
0,76 & $\mathrm{Wh} / \mathrm{kg} \cdot \mathrm{K}$ \\
18,63 & $\mathrm{~m}^{3}$ \\
0,16 & $\mathrm{~W} / \mathrm{m} \cdot \mathrm{K}$ \\
120 & $\mathrm{~kg} / \mathrm{m}^{3}$ \\
0,25 & $\mathrm{~m}$ \\
0,55 & $\mathrm{Wh} / \mathrm{kg} \cdot \mathrm{K}$ \\
0,045 & $\mathrm{~W} / \mathrm{m} \cdot \mathrm{K}$ \\
80 & $\mathrm{~kg} / \mathrm{m} 3$ \\
0,419 & $\mathrm{Wh} / \mathrm{kg} \cdot \mathrm{K}$ \\
0,042 & $\mathrm{~W} / \mathrm{m} \cdot \mathrm{K}$ \\
\hline
\end{tabular}

\section{Résultats}

La Figure7 donne la variation de la température à l'intérieur du grenier au cours d'une journée d'une part en prenant en compte un toit en tôle sans isolation, d'autre part, un toit en chaume.

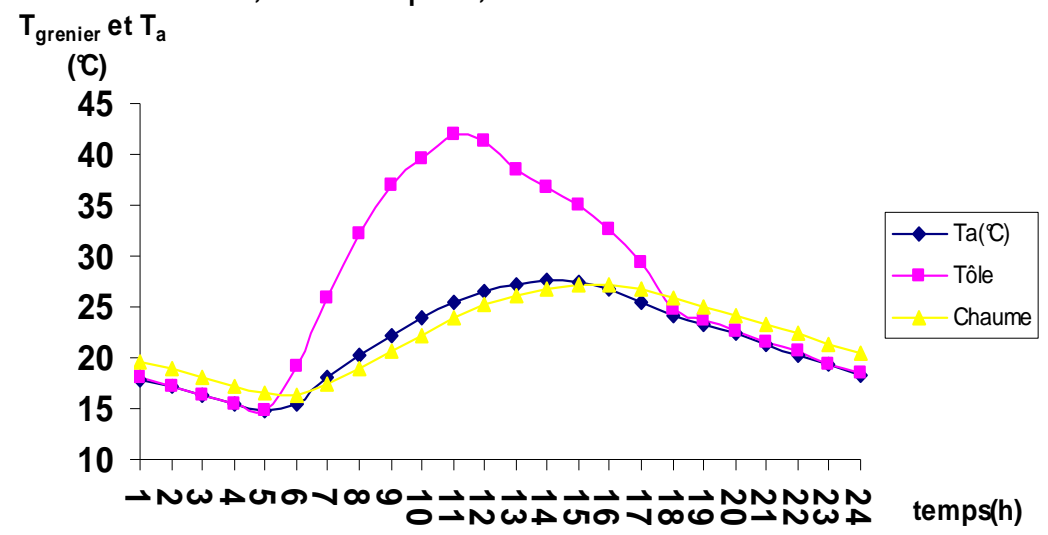

Figure 7: Comparaison du toit en tôle et du toit en chaume sans ventilation et sans isolation thermique selon la température du grenier. 
La Figure 8 donne la variation de la température à l'intérieur du grenier au cours d'une journée pour un toit en tôle en pratiquant la ventilation.

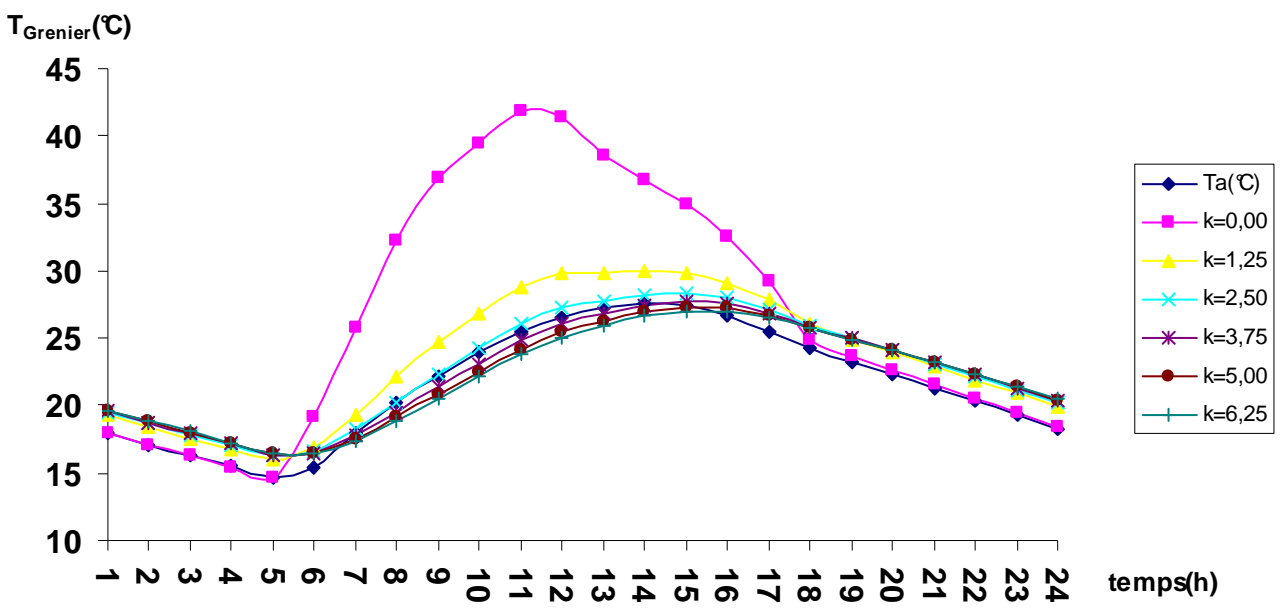

Figure 8 : Effet de la ventilation

La Figure 9 donne la variation de la température à l'intérieur du grenier au cours d'une journée pour un toit en tôle en pratiquant l'isolation.

La Figure 10 résume les sens des flux thermiques pour les deux toitures étudiées en fonction de l'heure de la journée. Les flux calculés pour le toit de chaume sont beaucoup plus faibles que ceux calculés pour le toit en tôle.

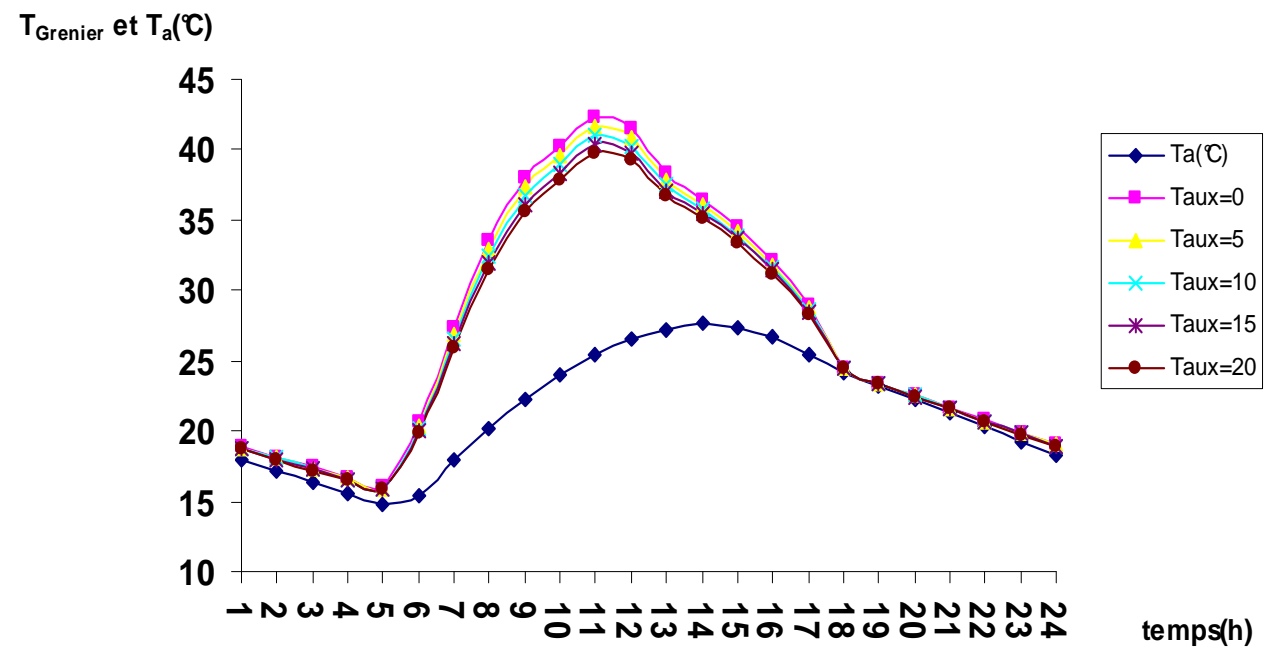

Figure 9 : Effet de l'isolation 

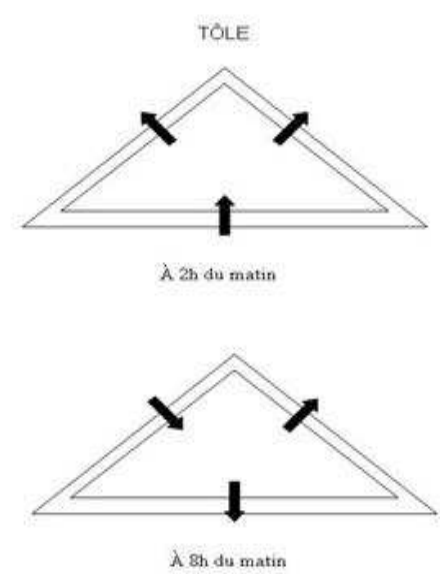

TOLE

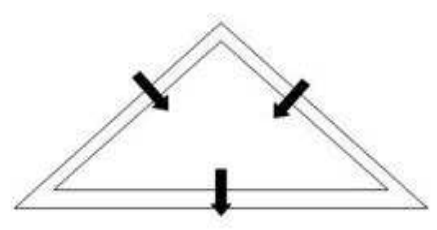

A 1 ih du matin

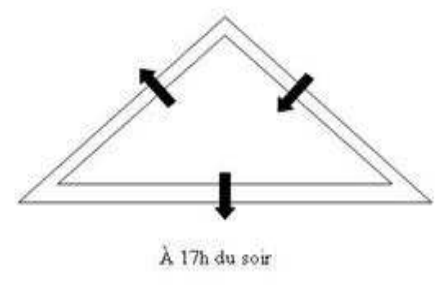

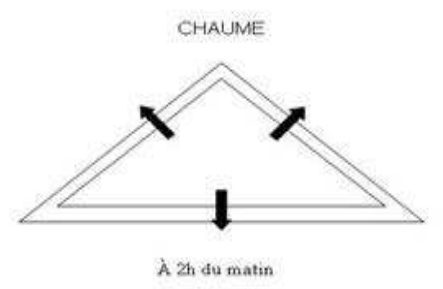

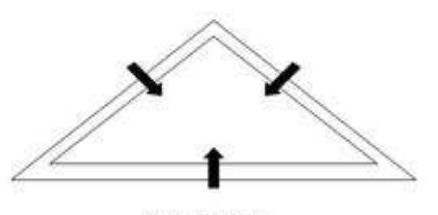

$\lambda$ gh du matin

CHAUME

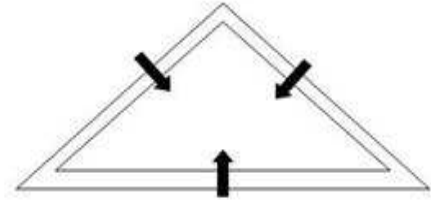

A $11 \mathrm{~h}$ du matin

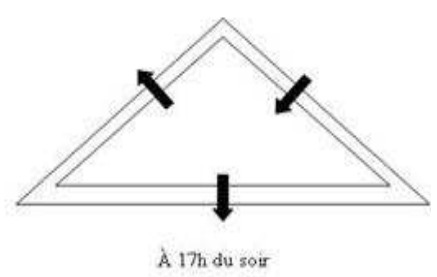

Figure 10: Sens de propagation des flux

\section{Discussion}

4-1. Comparaison de résultats entre le toit en tôle et le toit en chaume sans isolation et sans ventilation

- De la figure 7 , la nuit, de $18 \mathrm{~h}$ à $5 \mathrm{~h}$ du matin, la température du grenier est presque égale à celle de l'air extérieur pour les deux types de couverture. Par contre entre $6 \mathrm{~h}$ et $18 \mathrm{~h}$, en période ensoleillée, cette température est très supérieure à celle du milieu 
extérieur dans le cas du toit en tôle. La chaume étant un excellent isolant, la température du toit suit les variations de la température de l'air. La nuit le grenier est même un peu plus chaud et la journée un peu plus frais que l'air extérieur.

- En conclusion, ces résultats confirment les qualités thermiques du chaume par rapport à la tôle (figure 10). Cependant, les qualités d'étanchéité de la tôle ainsi que son coût moindre restent des raisons importantes et irréfutables de remplacement des toits traditionnels par de la tôle. II s'agit donc d'étudier des dispositifs pour minimiser au maximum cette dégradation du confort thermique. Deux solutions ont été étudiées: une ventilation du grenier et la mise en place d'un isolant interne.

\section{4-2. Ventilation du grenier pour un toit en tôle}

Nous imaginons de faire circuler de l'air dans le grenier à l'aide de deux ventilateurs, l'un au Sud et l'autre au Nord, afin de diminuer la température de l'air dans le grenier ( $T_{\text {Grenier }}$ ) pendant la période ensoleillée de la journée. La figure 8 donne la variation de TGrenier pendant une journée en fonction du taux de renouvellement d'air $\tau ; 0 \leq \tau \leq 20$.

Remarque : $\tau=0$ correspond à l'absence de ventilation.

Les différentes courbes ne se détachent que très peu malgré la grande variation du taux de renouvellement d'air de 0 à 20. Par exemple à $11 \mathrm{~h}$, la température du grenier ne diminue que de $2,5^{\circ} \mathrm{C}$ quand le taux de ventilation varie de 0 à 20 . Même si nous augmentons le taux de renouvellement d'air, c'est-à-dire le débit d'air, la température du grenier ne diminue que très peu pendant le jour. La ventilation n'est donc pas une bonne solution à envisager pour le rafraîchissement du bâtiment pour le toit en tôle. 


\section{4-3. Isolation du toit en tôle}

Etudions l'influence d'un isolant thermique en face inférieure de la tôle. La figure 9 donne les variations thermiques de l'air du grenier ( $T_{\text {Grenier}}$ en fonction de la qualité de cette isolation définie par: $k=\frac{e_{1}}{\lambda_{1}}$ avec $0,00 \leq k \leq 6,25$;

$k$ est alors le rapport de l'épaisseur $e_{1}$ de l'isolant par sa conductivité thermique $\lambda_{1}: k=\frac{e_{1}}{\lambda_{1}}$

Remarque : $k=0$ correspond au toit sans isolation thermique i. e. toit en tôle nue.

Les courbes s'approchent de celle du milieu ambiant quand $\mathrm{k}$ augmente. Une augmentation de valeur de $\mathrm{k}$ de 0,00 à $2,50 \mathrm{~m}^{2} . \mathrm{K} / \mathrm{W}$ seulement provoque une diminution de température de $16^{\circ} \mathrm{C}$. Cela veut dire que si nous utilisons le chaume dont le coefficient de conductibilité thermique étant égal à $0,045 \mathrm{~W} / \mathrm{m} . \mathrm{K}$, comme isolant, une épaisseur de $11,25 \mathrm{~cm}$ de chaume sous le toit en tôle suffit pour avoir cette diminution de température.

En conclusion, il suffit d'adjoindre un isolant en face intérieure de la tôle pour améliorer de manière très significative la qualité thermique de rafraîchissement.

\section{Conclusion}

Actuellement, la mise en place de la chaume sous la tôle sur les toits des maisons entraîne une dégradation extrêmement importante du confort thermique durant les périodes chaudes et ensoleillées. Cette dégradation a pu être quantifié grâce au modèle développé.

Ce modèle permet également de tester des solutions pour améliorer le confort thermique. L'ajout d'un isolant en face intérieure de la tôle semble 
une excellente solution. Le modèle devrait permettre de dimensionner l'épaisseur de cet isolant.

La suite de l'étude consistera à améliorer le modèle en précisant la valeur de certains paramètres. Par ailleurs, des mesures expérimentales seront nécessaires pour valider les calculs.

Enfin il faudra envisager de modéliser la maison dans son ensemble dans le but d'améliorer le confort thermique.

\section{Références}

[1] - Frédéric Miranville. "Contribution à l'étude des parois complexes en Physique du Bâtiment: Modélisation, Expérimentation et Validation Expérimentale de Complexes Toitures incluant des Produits Minces Réfléchissant en Climat Tropical Humide ". Thèse présentée devant l'Université de La Réunion pour obtenir le grade de Docteur. Spécialité: Génie Civil et Physique du Bâtiment. (2002).

[2] - H. RIETCSCHEL, W. RAISS. Traité de chauffage et de climatisation. Tome 1. Dunod. Traduction autorisée de l'ouvrage publié en langue allemande sous le titre : Heiz und klimatechnik Tome 1 par Springer - Verlag, Berlin et Heidelberg, New - York. 1968. Dunod, Paris, 1973 pour la traduction française. ISBN 2 - $04-004146-X$.

[3] - J. M. Chassériaux. Dunod. "Conversion thermique du rayonnement solaire ». A. F M. E Bordas, Paris, (1984) ISBN 2 $04-015601-1$.

[4] - RANAIVO RABEHAJA Bienvenue. "Caractéristiques du rayonnement solaire dans l'eau. Université d'Antananarivo". Mémoire de DEA d'Energétique (1983)

[5]- RALPH M. LEBENS. Habitat solaire passif. Méthodes de conception et de calcul.. de l'anglais par Michel PARIZEL. Paris, (1983).

[6] - RAJAONARIVELO Jeannot Arsène. Rapport de stage pour la préparation du Diplôme d'Université en Maîtrise et de 
l'Environnement. "Conception thermique de bâtiments monozones " Proposition de choix multiples des modèles. (1999).

[7] - Traité de Physique du bâtiment. Tome 1. Connaissances de base. CSTB le futur en construction. (1995). CSTB : Centre Scientifique et Technique du Bâtiment.

[8] - Association La Maison en Paille Logo La Maison en Paille - Stages \& Documentation · Entrée · dicton. (c) La Maison en Paille, Champmillon, France Résolution $800 * 600$.

www.lamaisonenpaille.com/ - $5 \mathrm{k}$ - En cache - Pages similaires

[9] - La-Maison-En-Paille

Nous souhaitons vous informer sur les activités de l'association et sur la construction en paille en général. Vous y trouverez entre autre:

www.lamaisonenpaille.com/1024x800/frame.htm - 2k - En cache $\underline{\text { Pages similaires }}$ 